\title{
Alternating-move hotelling with demand shocks
}

Citation for published version (APA):

Leufkens, K., \& Peeters, R. J. A. P. (2006). Alternating-move hotelling with demand shocks. METEOR, Maastricht University School of Business and Economics. METEOR Research Memorandum No. 039 https://doi.org/10.26481/umamet.2006039

Document status and date:

Published: 01/01/2006

DOI:

10.26481/umamet.2006039

Document Version:

Publisher's PDF, also known as Version of record

\section{Please check the document version of this publication:}

- A submitted manuscript is the version of the article upon submission and before peer-review. There can be important differences between the submitted version and the official published version of record.

People interested in the research are advised to contact the author for the final version of the publication, or visit the DOI to the publisher's website.

- The final author version and the galley proof are versions of the publication after peer review.

- The final published version features the final layout of the paper including the volume, issue and page numbers.

Link to publication

\footnotetext{
General rights rights.

- You may freely distribute the URL identifying the publication in the public portal. please follow below link for the End User Agreement:

www.umlib.nl/taverne-license

Take down policy

If you believe that this document breaches copyright please contact us at:

repository@maastrichtuniversity.nl

providing details and we will investigate your claim.
}

Copyright and moral rights for the publications made accessible in the public portal are retained by the authors and/or other copyright owners and it is a condition of accessing publications that users recognise and abide by the legal requirements associated with these

- Users may download and print one copy of any publication from the public portal for the purpose of private study or research.

- You may not further distribute the material or use it for any profit-making activity or commercial gain

If the publication is distributed under the terms of Article $25 \mathrm{fa}$ of the Dutch Copyright Act, indicated by the "Taverne" license above, 
Kasper Leufkens, Ronald Peeters

Alternating-move Hotelling with Demand Shocks

$\mathrm{RM} / 06 / 039$

JEL code: C61, C62, C72, C73, D43, L13

\section{METE@R}

Maastricht research school of Economics

of TEchnology and ORganizations

Universiteit Maastricht

Faculty of Economics and Business Administration P.O. Box 616

NL - 6200 MD Maastricht

phone : ++3143388 3830

fax $\quad$ : ++31433884873 



\title{
Alternating-move Hotelling with Demand Shocks
}

\author{
Kasper Leufkens* $\quad$ Ronald Peeters*
}

September 5, 2006

\begin{abstract}
In this paper an infinite-horizon alternating-move Hotelling model in which consumers are uniformly distributed over the market is considered. In a Markov perfect equilibrium, a seller's move in any period depends on the price the other seller is committed to. The analytic solution is given and the unique linear Markov perfect equilibrium is computed for different values of the discount factor. The base model is then extended by the introduction of exogenous demand shocks which makes finding an analytical solution using the conventional analysis impossible. For this extended model the margin in which long-run prices fluctuate is determined for different values of the shock probability. It is found that the prices set in the high demand state are always lower than in the low demand state.
\end{abstract}

JEL Classification Codes: C61, C62, C72, C73, D43, L13.

Keywords: Hotelling model, Alternating-move model, Markov perfect equilibrium.

\section{Introduction}

When a competitive situation is modeled in a static setting, firms are found to behave too noncooperative due to a lack of opportunities to reward cooperative behavior and/or to punish aggressive competitive behavior. When the scenario of being able to drive ones opponent out of the market is unrealistic, firms are bound to allow each other in the market. If they then compete heavily this comes at the expense of all firms' profits. Therefore it seems plausible that firms will act mutually nonaggressive in order to ensure higher profits to all of them (including themselves). In order to be able to obtain results that capture mutual nonaggressive behavior dynamic models have to be considered.

This paper presents a model with two sellers located at the end points of a linear city that is uniformly distributed by potential customers and where the two sellers alternately set prices. The alternate decision making captures the idea of short-run price commitments. The sellers' strategies are assumed to depend on the physical state of the system, which is in the present setting simply the price the other seller is currently committed to. Therefore strategies are dynamic reaction functions that give for each price, a price in response. A pair

${ }^{*}$ Department of Economics, Universiteit Maastricht, P.O. Box 616, 6200 MD, Maastricht, The Netherlands, e-mail: \{K.Leufkens, R.Peeters\}@algec.unimaas.nl. The second author is financially supported by NWO. 
of dynamic reaction functions constitutes a Markov perfect equilibrium (MPE) if this pair forms a (subgame perfect) equilibrium.

For the model under consideration we compute the unique linear MPE. This MPE is found to be dynamically stable as the dynamic reactions converge to a steady state. The MPE is found numerically by applying backward induction after truncation of the horizon. By using similar arguments as in Maskin and Tirole (1987) the finite horizon equilibrium strategies converge to the unique linear MPE of the infinite-horizon model if the truncation of the horizon lengthens.

The literature on alternating-move models started with the pioneering contribution of $\mathrm{Cy}$ ert and DeGroot (1970) who analyzed an alternating-move duopoly model where the strategic variable is quantity. Although they consider a finite horizon they pay attention to the longrun price when the horizon is far. Maskin and Tirole (1987) considers the infinite-horizon version and shows via a contraction mapping argument that the finite-horizon model of Cyert and DeGroot (1970) converges to their solution if the truncation of the horizon lengthens.

Maskin and Tirole (1988a) considers an infinite-horizon alternating-move model of quantity competition in a homogeneous product market with fixed costs that large that only one firm is able to make a profit. It is found that in the unique MPE only one firm is active and practices the quantity analogue of limit pricing. Maskin and Tirole (1988b) considers a comparable model with homogeneous products where competition is in prices instead of quantities. It is concluded that an increase in the discount factor makes it more worthwhile for a firm to sacrifice current clientele by raising its price today in the expectation of future profit when the other firm follows suit.

Eaton and Engers (1990) considers a similar model as Maskin and Tirole (1988b) but then in a differentiated product market. For this model two kind of equilibria are found: a 'disciplined' one in which the steady state is enforced by threats to undercut and that arises when the products are close substitutes, and a 'spontaneous' one in which such threats are not needed and that arises when the products are more differentiated. The main difference with the present study is that Eaton and Engers (1990) considers a linear city where half of the consumers is located at one of the endpoints and the other half at the other endpoint. The present paper considers a situation with more heterogeneity among consumers. From a strategic perspective the models are completely different as the concept of 'undercutting' has no sensible meaning in the present setting.

De Fraja (1993) considers a model where unions care about wages and employment. It is found that the long-run equilibrium wage level is higher when the bargaining process occurs sequentially than when it happens simultaneously.

Like in the present paper, Rath (1998) examines an infinite-horizon Hotelling model with linear transportation costs. But, after the firms have chosen location they repeatedly set prices simultaneously. Firms have to resort to 'carrot-and-stick' strategies in order to sustain the cooperative outcome with prices above the base level from the static model. 
Baye and Ueng (1999) considers a model similar to Maskin and Tirole (1987) but with a differentiated products price setting environment. Closed-form solutions for the Markov perfect equilibrium prices are given for linear demand. It is found that in equilibrium prices are strictly higher than the one-shot Nash equilibrium prices, but lower than fully collusive prices.

Finally, Eckert (2004) extends Maskin and Tirole (1988b) by examining price matching strategies when marginal costs vary over time. It is shown that provided marginal costs do not fluctuate excessively, equilibria in which firms match the current monopoly price do not exist when the probability that the marginal cost remain in their current state is low. However, focal price equilibria still exist. A similar result is found for fluctuating demand and constant marginal costs.

Besides the mentioned model differences with the existing literature, the present paper generally differs in approach. Here the focus will not be on specific tacit collusion equilibria but on normal MPE. Moreover, this paper extends the model by introducing exogenous demand shocks. There are two demand levels (high and low) and given the current demand level there is a probability that the demand level changes in the next period. In this setting, each seller has two dynamic reaction functions: one in case the demand is high and one in case the demand is low. The introduction of uncertainty into the model has fundamental consequences as the techniques used in Maskin and Tirole (1987) can no longer be applied. Therefore the MPE is computed numerically by applying backward induction after truncating the horizon. We determine the margin in which long-run prices fluctuate and the dependency of this margin on the shock probability and the discount factor.

The paper is organized as follows. In the next section the static model and its solution is presented. Section 3 contains the alternating-move variant of this model. In Section 4 the dynamic reaction functions that constitute a Markov perfect equilibrium are determined. The reaction functions are computed numerically and the movement of prices in the long-run are discussed. In Section 5 the model is extended by including exogenous demand shocks. Section 6 concludes.

\section{$2 \quad$ Hotelling model}

The simplest model of horizontal differentiation is given by Hotelling in 1929. Consider a linear beach with two ice cream vendors that are located at the two ends of a beach both selling the same brand of ice cream. The seaside visitors, that are the potential customers, are uniformly distributed over the beach. Dependent on the prices the vendors ask for their ice cream, each visitor decides to which vendor to go, taking into account the transportation cost over the distance from their blanket to the chosen vendor. A more general interpretation of this model is that consumers' preferences are uniformly distributed over the interval and that each consumer receives an increasing disutility the further the product is away from his 
most preferred option.

Let the beach be represented by the unit interval $[0,1]$ and let vendor $A$ be located at $x=0$ and vendor $B$ at $x=1$. Moreover, let each visitor's gross surplus from consuming ice cream be equal to $\beta$ and let the transportation costs per unit of distance be equal to $\tau$. Throughout the whole paper we assume that $\beta$ is sufficiently large such that the market will be served completely. Finally, we assume that production (exploitation) costs are zero.

The situation described above is a two-stage model, where in the first stage both vendors determine prices and in the second stage the seaside visitors make their decisions. This model can be solved by backwards induction.

In stage 2 , given prices $p^{A}$ and $p^{B}$, the visitor located at $x$ has a utility of

$$
U^{x}= \begin{cases}0 & \text { if the visitor doesn't consume ice cream; } \\ \beta-p^{A}-\tau \cdot x & \text { if the visitor consumes the ice cream at vendor } A \\ \beta-p^{B}-\tau \cdot(1-x) & \text { if the visitor consumer the ice cream at vendor } B\end{cases}
$$

The indifferent visitor is then located at

$$
\hat{x}=\frac{1}{2}+\frac{p^{B}-p^{A}}{2 \tau} .
$$

So, given the prices $p^{A}$ and $p^{B}$, vendor $A$ attracts $q^{A}=\hat{x}$ visitors and vendor $B$ the remaining $q^{B}=1-\hat{x}$ visitors.

In stage 1 , knowing the reaction of the potential customers, both vendors simultaneously decide on prices with the purpose to maximize their profit $\pi^{i}=q^{i} p^{i}(i=A, B)$ and anticipating on the price the opponent might (and will) set. Given $p^{B}$, vendor $A$ 's profit is maximized by setting $p^{A}=\frac{p^{B}+\tau}{2}$; given $p^{A}$, vendor $B$ 's profit is maximized by setting $p^{B}=\frac{p^{A}+\tau}{2}$. As in equilibrium both vendors are optimally responding against one another, both equations have to be satisfied, leading to the equilibrium prices, quantities and profits:

$$
p^{A *}=p^{B *}=\tau, \quad q^{A *}=q^{B *}=\frac{1}{2} \quad \text { and } \quad \pi^{A *}=\pi^{B *}=\frac{1}{2} \tau .
$$

Although this solution might give a good prediction for market prices when the vendors set their prices once, in reality the competition is less static and the vendors are able to react on each other's prices. In order to capture this dynamic feature of reality the next section presents an infinite-horizon alternating-move variant of the Hotelling model.

\section{Alternating-move Hotelling model}

In the infinite-horizon alternating-move variant of the Hotelling model from the previous section, the ice cream vendors are able to react on prices previously set by the opponent in an alternating manner. Without loss of generality we assume that vendor $A$ is the first to act.

In the first stage, given a pair of initial prices $\left(p_{0}^{A}, p_{0}^{B}\right)$, vendor $A$ sets a price $p_{1}^{A}$ in order to compete against vendor $B$ 's price $p_{1}^{B}:=p_{0}^{B}$. This leads to instantaneous profits $\pi^{A}\left(p_{1}^{A}, p_{1}^{B}\right)$ and $\pi^{B}\left(p_{1}^{A}, p_{1}^{B}\right)$ for the vendors. But besides determining the instantaneous profits in the 
current stage, vendor $A$ immediately determines its second stage price as it is assumed that the vendors are only able to adjust prices every second stage.

Then, in the second stage, given vendor $A$ 's price set in the first stage $p_{2}^{A}:=p_{1}^{A}$, vendor $B$ has to set a price $p_{2}^{B}$ in order to obtain instantaneous profits and influence dynamics by immediately having determined its third stage's price. This procedure is infinitely repeated. That is, in odd stages $k$ vendor $A$ sets a price $p_{k}^{A}$ against the price $p_{k}^{B}:=p_{k-1}^{B}$ that vendor $B$ has set in the previous stage and in even stages $k$ vendor $B$ sets a price $p_{k}^{B}$ against the price $p_{k}^{A}:=p_{k-1}^{A}$ that vendor $A$ has set in the previous stage.

Both vendors' goal is to maximize the present value of the complete stream of profits by application of the discount factor $\delta \in(0,1)$. So, in each stage in which a vendor can set a price it does not only take into account the current profits but also the possible reaction of the opponent in the next stage and its own reaction on that and so on.

A strategy for a vendor is a specification of its behavior dependent on time and all prices set in previous rounds. Quite naturally, a pair of strategies constitutes an equilibrium if no vendor is able to improve the present value of the stream of profits by a unilateral (and one-shot) deviation. A strategy is called stationary if it is independent of time and history and consequently depends only on the current state. In this model the current state is the price the opponent set in the previous stage. In a stationary strategy, vendor $A$ specifies for each possible price $p^{B}$ of vendor $B$ its optimal response $p^{A}\left(p^{B}\right)$. Every time when vendor $B$ decides on the price $p^{B}$, in the next round vendor $A$ will react with $p^{A}\left(p^{B}\right)$ regardless of time or history of prices. Therefore, stationary strategies can be seen as dynamic reaction functions. Once a pair of such stationary strategies $\left(p^{A}(\cdot), p^{B}(\cdot)\right)$ constitutes an equilibrium, this equilibrium is called a Markov perfect equilibrium (MPE).

In the remaining of this paper, analysis is restricted to the use of stationary strategies. Several technical and pragmatic motivations for this restriction can be found in Maskin and Tirole (2001). An experimental motivation for this restriction can be found in McKelvey and Palfrey (1995). The next section is devoted to the analysis of the alternating-move model by computing the unique linear MPE and reporting on its properties.

\section{Markov perfect equilibrium and price dynamics}

Theorem 4.1 For any discount factor $\delta$ there exists a unique linear MPE.

Proof See Theorem A.2 and its proof in the appendix.

A way to compute the stationary equilibrium is by gradually truncation of the horizon and applying backwards induction to the truncated games (Maskin and Tirole, 1987 and Lau, 2002). In all maximization problems during the backwards induction procedure the objective function is quadratic in the decision variable. Therefore, the only MPE equilibrium that can be found by applying the backwards induction procedure is (the unique) one with linear 
dynamic reaction functions. For different values of the discount factor $\delta$, Table 1 displays the symmetric unique linear MPE strategies of the two vendors that are found in this way.

\begin{tabular}{c|ccc|c}
$\delta$ & $p^{i *}\left(p^{j}\right)$ & & $p^{i *}\left(p^{j}\right)$ \\
\hline 0.00 & $0.500000 \cdot p^{j}+0.500000 \cdot \tau$ & & 0.50 & $0.384410 \cdot p^{j}+0.762062 \cdot \tau$ \\
0.05 & $0.487578 \cdot p^{j}+0.525226 \cdot \tau$ & & 0.55 & $0.374173 \cdot p^{j}+0.787992 \cdot \tau$ \\
0.10 & $0.475316 \cdot p^{j}+0.550867 \cdot \tau$ & & 0.60 & $0.364228 \cdot p^{j}+0.813566 \cdot \tau$ \\
0.15 & $0.463221 \cdot p^{j}+0.576861 \cdot \tau$ & 0.65 & $0.354584 \cdot p^{j}+0.838725 \cdot \tau$ \\
0.20 & $0.451304 \cdot p^{j}+0.603136 \cdot \tau$ & 0.70 & $0.345245 \cdot p^{j}+0.863419 \cdot \tau$ \\
0.25 & $0.439579 \cdot p^{j}+0.629612 \cdot \tau$ & 0.75 & $0.336213 \cdot p^{j}+0.887605 \cdot \tau$ \\
0.30 & $0.428063 \cdot p^{j}+0.656206 \cdot \tau$ & 0.80 & $0.327489 \cdot p^{j}+0.911251 \cdot \tau$ \\
0.35 & $0.416772 \cdot p^{j}+0.682833 \cdot \tau$ & 0.85 & $0.319070 \cdot p^{j}+0.934329 \cdot \tau$ \\
0.40 & $0.405723 \cdot p^{j}+0.709406 \cdot \tau$ & 0.90 & $0.310952 \cdot p^{j}+0.956821 \cdot \tau$ \\
0.45 & $0.394931 \cdot p^{j}+0.735842 \cdot \tau$ & 0.95 & $0.303130 \cdot p^{j}+0.978714 \cdot \tau$
\end{tabular}

Table 1: Markov perfect equilibrium strategies

When $\delta$ equals zero, the dynamic reaction functions coincide with their static counterparts given in Section 2. If $\delta$ converges to 1 , these linear dynamic reaction functions converge to

$$
p^{i *}\left(p^{j}\right)=0.295598 p^{j}+1.000000 \tau .
$$

The decrease in the coefficients in front of $p^{j}$ for increasing values of $\delta$ indicates that the reactivity on each other's prices is less when the vendors are more patient. The increase in the coefficients in front of $\tau$ for increasing values of $\delta$ indicates that the vendors' willingness to coordinate on a higher price increases when the vendors are more patient.

In Figure 1, impressions of the shape of the equilibrium strategies (linear dynamic reaction functions) are drawn. Although the lines in the figure have a similar shape as the best-response curves of the static model, they do not represent best-response curves. Given that vendor $B$ plays the stationary strategy $p^{B *}(\cdot)$, the whole line $p^{A *}(\cdot)$ is the best response for vendor $A$ and $p^{A *}\left(p^{B}\right)$ is just the present stage's price realization when in the previous stage vendor $B$ has set the price $p^{B}$. So, the only best response drawn for vendor $A$ in this figure, is the best response against vendor $B$ 's strategy $p^{B *}(\cdot){ }^{1}$

Let the initial state $\left(p_{0}^{A}, p_{0}^{B}\right)$ be given by point 0 in the figure. In the first stage vendor $A$ will face the price $p_{1}^{B}:=p_{0}^{B}$ and react with $p_{1}^{A}=p^{A *}\left(p_{1}^{B}\right)$, which leads to the price pair $\left(p_{1}^{A}, p_{1}^{B}\right)$ in point 1 . In the next stage vendor $A$ is committed to its first stage price: $p_{2}^{A}:=p_{1}^{A}$. Given the state $p_{2}^{A}$, vendor $B$ 's decision as specified by its stationary strategy $p^{B *}(\cdot)$ is $p_{2}^{B}=p^{B *}\left(p_{2}^{A}\right)$, which brings the system to point 2 . Repeating this procedure we see that the state dynamics converges to one single point where both vendors apply the steady-state price $\bar{p}$.

Theorem 4.2 For any discount factor $\delta$, the MPE is dynamically stable. Each vendor's steady state price is equal to $\bar{p}=\frac{b}{1-a} \tau$, where $b \tau$ is the intercept and a the slope of the Markov perfect equilibrium strategy.

\footnotetext{
${ }^{1}$ Cyert and DeGroot (1970) state that in the best-response dynamics of the static model the assumption underlies that the rival will not change his decision in response to a change by the firm. This assumption is proved false at each period, but firms continue to use reaction functions that are based on the false assumption.
} 


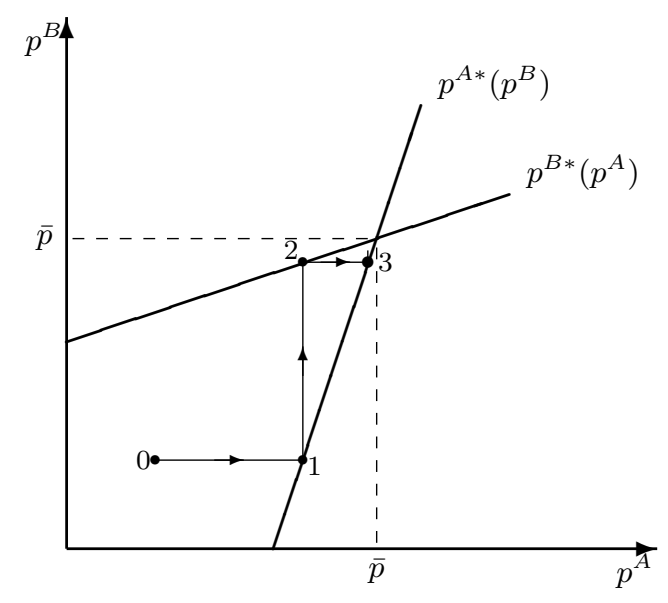

Figure 1: MPE strategies

Proof See Theorem A.2 and its proof in the appendix.

Regardless of the price-pair $\left(p_{0}^{A}, p_{0}^{B}\right)$ in which the procedure starts, the price dynamics converges to the point $(\bar{p}, \bar{p})$. Hence, in the long-run prices converge to $\bar{p}$. For different values of $\delta$, Table 2 lists the values of the corresponding long-run price and is graphically supported by Figure 2 (for $\tau=1$ ). The long-run price can be computed to converge to $\bar{p}=1.419643 \cdot \tau$ when $\delta$ converges to 1 .

\begin{tabular}{c|c}
$\delta$ & $\bar{p}$ \\
\hline 0.00 & $1.000000 \cdot \tau$ \\
0.05 & $1.024988 \cdot \tau$ \\
0.10 & $1.049904 \cdot \tau$ \\
0.15 & $1.074672 \cdot \tau$ \\
0.20 & $1.099216 \cdot \tau$ \\
0.25 & $1.123463 \cdot \tau$ \\
0.30 & $1.147340 \cdot \tau$ \\
0.35 & $1.170782 \cdot \tau$ \\
0.40 & $1.193729 \cdot \tau$ \\
0.45 & $1.216129 \cdot \tau$
\end{tabular}

\begin{tabular}{c|c}
$\delta$ & $\bar{p}$ \\
\hline 0.50 & $1.237938 \cdot \tau$ \\
0.55 & $1.259121 \cdot \tau$ \\
0.60 & $1.279651 \cdot \tau$ \\
0.65 & $1.299511 \cdot \tau$ \\
0.70 & $1.318690 \cdot \tau$ \\
0.75 & $1.337184 \cdot \tau$ \\
0.80 & $1.354997 \cdot \tau$ \\
0.85 & $1.372136 \cdot \tau$ \\
0.90 & $1.388612 \cdot \tau$ \\
0.95 & $1.404444 \cdot \tau$
\end{tabular}

Table 2: Long-run prices

Proposition 4.3 The steady state price $\bar{p}$ is equal to the static Hotelling solution for $\delta=0$, and grows with the discount factor.

The positive correlation between the discount factor and the long-run prices reveals that by increasing patience the vendors become mutually less aggressive. For any discount factor larger than zero, the long-run prices are higher than in the static model. 


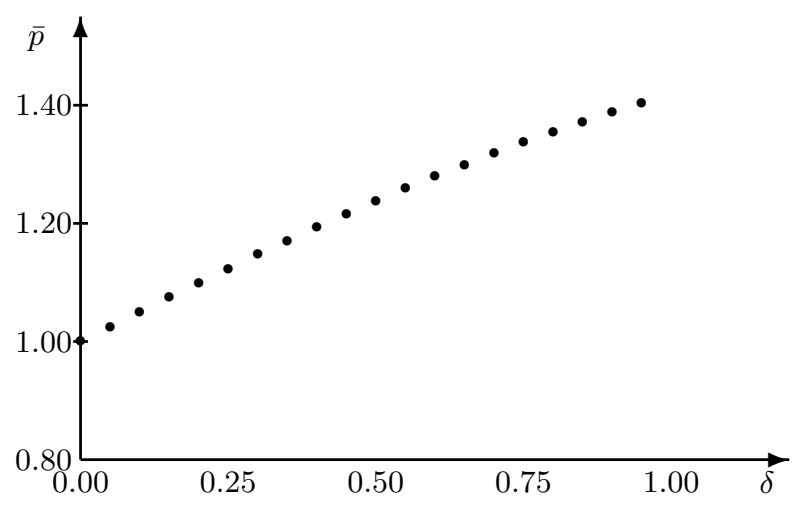

Figure 2: Long-run prices.

\section{$5 \quad$ Exogenous demand shocks}

In this section the alternating-move Hotelling model is extended by incorporating exogenous demand shocks. As already mentioned in the introduction, we then have to resort to numerical computations. Namely, in the analysis of the situation without demand shocks the reaction on the opponent's reaction on the own price set is used (see Maskin and Tirole (1987) or Equation (2) in the appendix). With demand shocks, however, the reaction of your opponent on your price cannot be perfectly anticipated, since it depends on the state of demand the opponent faces. Hence, the reaction on the opponent's reaction on the own price previously set is no longer well-defined.

Let there be two possible states of demand: low demand and high demand. Assume that in the high demand state the market is $h$ times the size of the market in the low demand state. Moreover, the probability of going from one state of demand to the other over periods is equal to $\alpha$. That is, if the process is currently in the low (high) demand state, the next period the demand will be high (low) with probability $\alpha$ and will stay low (high) with probability $1-\alpha$. For instance, the weather changes between sunny and cloudy with probability $\alpha$ (hence, tomorrow's weather is related to today's weather) and demand for ice creams is high when it is sunny.

In a stationary strategy for this setting, vendor $A$ specifies a pair $\left(p^{A}\left(H, p^{B}\right), p^{A}\left(L, p^{B}\right)\right)$ consisting of a price to respond with in case the demand is high and a price to respond with in case the demand is low for each possible price $p^{B}$. So, a stationary strategy is a pair of dynamic reaction functions: one that is applied in case the demand is high and one that is applied in case the demand is low. Quite naturally, when a pair of stationary strategies $\left(\left(p^{A}(H, \cdot), p^{A}(L, \cdot)\right),\left(p^{B}(H, \cdot), p^{B}(L, \cdot)\right)\right)$ constitutes an equilibrium it is called a MPE.

For the remaining of this section the relative demand difference is fixed at $h=1.50$. Like in the situation without exogenous demand shocks, backwards induction is applied to solve for the MPE. For $\delta=0.95$ and different values of $\alpha$, Table 3 contains the symmetric linear 
MPE dynamic reaction functions (second and third column).

\begin{tabular}{c|c|c|c}
$\alpha$ & $p^{i *}\left(H, p^{j}\right)$ & $p^{i *}\left(L, p^{j}\right)$ & $\tilde{p}^{j}$ \\
\hline 0.00 & $0.303130 \cdot p^{j}+0.978714 \cdot \tau$ & $0.303130 \cdot p^{j}+0.978714 \cdot \tau$ & $\infty$ \\
0.10 & $0.307865 \cdot p^{j}+0.965787 \cdot \tau$ & $0.296352 \cdot p^{j}+0.997095 \cdot \tau$ & $2.719361 \cdot \tau$ \\
0.20 & $0.312373 \cdot p^{j}+0.954155 \cdot \tau$ & $0.290446 \cdot p^{j}+1.012004 \cdot \tau$ & $2.638254 \cdot \tau$ \\
0.30 & $0.316682 \cdot p^{j}+0.943610 \cdot \tau$ & $0.285230 \cdot p^{j}+1.024266 \cdot \tau$ & $2.564416 \cdot \tau$ \\
0.40 & $0.320817 \cdot p^{j}+0.934019 \cdot \tau$ & $0.280573 \cdot p^{j}+1.034417 \cdot \tau$ & $2.494732 \cdot \tau$ \\
0.50 & $0.324797 \cdot p^{j}+0.925291 \cdot \tau$ & $0.276375 \cdot p^{j}+1.042831 \cdot \tau$ & $2.427409 \cdot \tau$ \\
0.60 & $0.328638 \cdot p^{j}+0.917369 \cdot \tau$ & $0.272559 \cdot p^{j}+1.049774 \cdot \tau$ & $2.361205 \cdot \tau$ \\
0.70 & $0.332357 \cdot p^{j}+0.910180 \cdot \tau$ & $0.269065 \cdot p^{j}+1.055441 \cdot \tau$ & $2.295093 \cdot \tau$ \\
0.80 & $0.335965 \cdot p^{j}+0.903716 \cdot \tau$ & $0.265843 \cdot p^{j}+1.059976 \cdot \tau$ & $2.228402 \cdot \tau$ \\
0.90 & $0.339475 \cdot p^{j}+0.897945 \cdot \tau$ & $0.262856 \cdot p^{j}+1.063485 \cdot \tau$ & $2.160561 \cdot \tau$ \\
1.00 & $0.342898 \cdot p^{j}+0.892857 \cdot \tau$ & $0.260068 \cdot p^{j}+1.066042 \cdot \tau$ & $2.090849 \cdot \tau$
\end{tabular}

Table 3: Markov perfect equilibrium strategies

When $\alpha=0.00$ the situation is equivalent to the situation without exogenous demand shocks. As a result, similar dynamic reaction functions are found and consequently the longrun prices will converge to $\bar{p}=1.404444 \tau$. The long-run prices are independent of the state of demand as in both states of demand the situation in which the vendors manifest are strategically equivalent.

When $\alpha=1.00$ the situation is in the extreme case of alternating states of demand. That is, when the current demand is low the next period the demand will be high for sure, and vice versa. In this extreme scenario the symmetric linear MPE dynamic response functions will lead to prices to alternate between $\bar{p}(H)=1.381609 \tau$ and $\bar{p}(L)=1.425354 \tau$ in the long-run. Note that in the high state the price is lower than in the low state and that the price without demand shocks is in between both.

For all values of $\alpha$ the MPE dynamic reaction functions in the table are of the structure $p^{i *}\left(H, p^{j}\right)=a_{H} p^{j}+b_{H} \tau$ and $p^{i *}\left(L, p^{j}\right)=a_{L} p^{j}+b_{L} \tau$. When $\alpha=0.00$, then $a_{H}=a_{L}$ and $b_{H}=b_{L}$. In the high state the reactivity on each others prices, $a_{H}$, increases the more likely a switch is. The decreasing $b_{H}$ signals a lower willingness to coordinate on high prices. In the low state exactly the opposite is observed.

For given $p^{j}, p^{i *}\left(L, p^{j}\right) \geq p^{i *}\left(H, p^{j}\right)$ if and only if $p^{j} \leq \tilde{p}^{j} \equiv \frac{b_{L}-b_{H}}{a_{H}-a_{L}} \tau$. For each $\alpha$ this latter value $\tilde{p}^{j}$ is shown in the fourth column of Table 3. For each value of $\alpha, \tilde{p}^{j}$ is larger than the long-run prices in the two extreme scenarios (no demand shocks and alternating demands). Therefore, it is likely that $p^{i *}\left(L, p^{j}\right) \geq p^{i *}\left(H, p^{j}\right)$ for all values of $\alpha$. This implies that vendors set higher prices when the demand is low than when the demand is high regardless of the probability of being in the high (low) demand when the current demand is low (high).

Suppose that, given the current price to which the opponent is committed, the vendors indeed set a higher price when the demand is low than when the demand is high. Then, given an initial price, always being in the high state must lead to the lowest long-run price possible. When the system is always in the high state, prices will continuously be determined 
by the dynamic reaction function $p^{i *}\left(H, p^{j}\right)=a_{H} p^{j}+b_{H} \tau$. As a consequence the prices will converge to $\hat{p}(H)=\frac{b_{H}}{1-a_{H}} \tau$ in such a case. Therefore the long-run prices must always be more than $\hat{p}(H)$. For similar reasons it can be concluded that long-run prices are always smaller than $\hat{p}(L)=\frac{b_{L}}{1-a_{L}} \tau$. The price bounds $\hat{p}(H)$ and $\hat{p}(L)$ are shown in the second and third column of Table 4 . As for all values of $\alpha$ both $\hat{p}(H)$ and $\hat{p}(L)$ are less than $\tilde{p}^{j}$, the supposition in the beginning of this paragraph — and hence the following proposition — is justified.

\begin{tabular}{c|c|c|c|c}
$\alpha$ & $\hat{p}(H)$ & $\hat{p}(L)$ & $\check{p}(H)$ & $\check{p}(L)$ \\
\hline 0.00 & $1.404444 \cdot \tau$ & $1.404444 \cdot \tau$ & $1.404444 \cdot \tau$ & $1.404444 \cdot \tau$ \\
0.10 & $1.395374 \cdot \tau$ & $1.417037 \cdot \tau$ & $1.402043 \cdot \tau$ & $1.410617 \cdot \tau$ \\
0.20 & $1.387605 \cdot \tau$ & $1.426254 \cdot \tau$ & $1.399678 \cdot \tau$ & $1.415028 \cdot \tau$ \\
0.30 & $1.380924 \cdot \tau$ & $1.433001 \cdot \tau$ & $1.397416 \cdot \tau$ & $1.418147 \cdot \tau$ \\
0.40 & $1.375210 \cdot \tau$ & $1.437835 \cdot \tau$ & $1.395301 \cdot \tau$ & $1.420264 \cdot \tau$ \\
0.50 & $1.370389 \cdot \tau$ & $1.441121 \cdot \tau$ & $1.393363 \cdot \tau$ & $1.421572 \cdot \tau$ \\
0.60 & $1.366416 \cdot \tau$ & $1.443105 \cdot \tau$ & $1.391628 \cdot \tau$ & $1.422207 \cdot \tau$ \\
0.70 & $1.363273 \cdot \tau$ & $1.443960 \cdot \tau$ & $1.390090 \cdot \tau$ & $1.422250 \cdot \tau$ \\
0.80 & $1.360946 \cdot \tau$ & $1.443800 \cdot \tau$ & $1.388782 \cdot \tau$ & $1.421774 \cdot \tau$ \\
0.90 & $1.359441 \cdot \tau$ & $1.442710 \cdot \tau$ & $1.387709 \cdot \tau$ & $1.420822 \cdot \tau$ \\
1.00 & $1.358780 \cdot \tau$ & $1.440730 \cdot \tau$ & $1.386880 \cdot \tau$ & $1.419417 \cdot \tau$
\end{tabular}

Table 4: Bounds on the long-run prices

Proposition 5.1 The response price set when demand is low is higher than when demand is high for each price the opponent is committed to.

The intuition behind this proposition is that when the demand is high, competition for the market gets fiercer, resulting in lower prices. Lowering the price will decrease the profit per consumer in both states. These forgone per unit profits are easier compensated for when the demand is high. Hence, response prices will be lower when the demand is high.

As mentioned before, $p^{i *}\left(H, p^{j}\right)$ increases in $p^{j}$ and $\hat{p}(L)$ is the maximum long-run price. Then for each $\alpha$ the maximum price that can be observed in the high state is $\check{p}(H) \equiv$ $p^{i *}(H, \hat{p}(L))$. Similarly $\check{p}(L) \equiv p^{i *}(L, \hat{p}(H))$ is the minimum price that can be observed in the low state. The price bounds $\check{p}(H)$ and $\check{p}(L)$ are shown in the fourth and fifth column of Table 4. It can be concluded that the long-run prices will be set between $\check{p}(H)$ and $\hat{p}(H)$ when demand is high and between $\hat{p}(L)$ and $\check{p}(L)$ when demand is low. These bounds are displayed in Figure 3.

The figure reveals that the lower bound on the prices in the low demand is always above the upper bound on the prices in the high demand. Extensive numerical computations have shown that this property is found to hold true for any value of $\delta$ and $h$ (with $h>1$ ).

Proposition 5.2 In the long-run, the prices set when demand is low are always higher than when demand is high.

Without demand shocks (that is, when $\alpha=0$ ) long-run prices are equal in both states of demand. In the presence of a positive shock probability, as already mentioned above, 


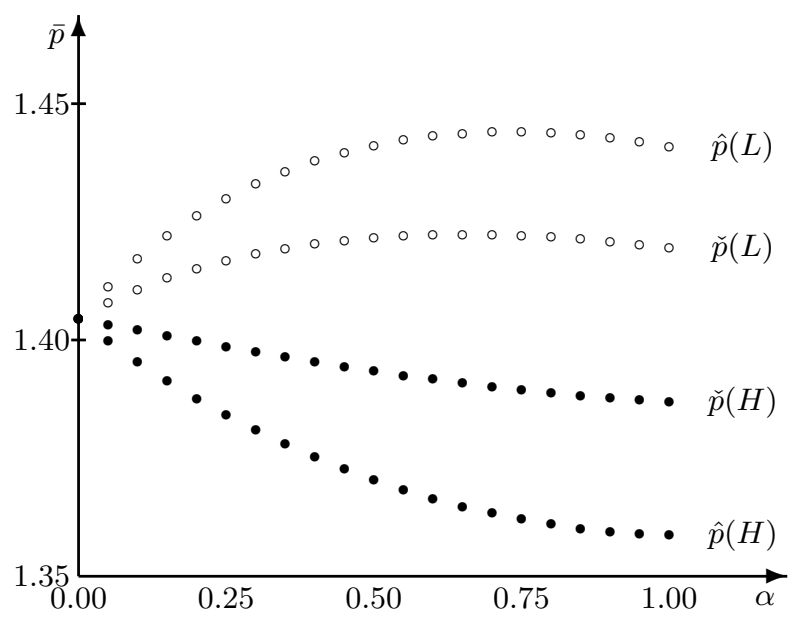

Figure 3: Bounds on long-run prices

competition is much fiercer in the high demand. In the figure we see a downward trend in the price-bounds in the high demand for increasing values of the shock probability. The reason is that when the probability to leave the high demand increases, the incentive to reap the immediate profits at stake becomes larger.

In the low demand we see that the price-bounds initially increase in the shock probability. The reason is that the probability of going to the high demand state becomes larger and getting there with a higher current price is more attractive. The vendor that sets the price in the low demand is giving up some of the immediate reward in order to benefit from it in the next stage. Once the shock probability reaches a certain level (in the figure at a point close to $\alpha=0.75)$ the price-bounds in the low demand start decreasing in the shock probability. The reason is that the probability of a return to the low demand after one stage of high demand is also increasing, resulting in a diminishing incentive to act nonaggressive in the low state. In the end, it is the opponent firm that will benefit from such leniency.

For low discount factors the influence of future periods is too small and the critical point in the price-bound in the low state is not observed. For larger values of $h$ the critical point is located at a lower value of $\alpha$.

\section{Conclusion}

In this paper an alternating-move Hotelling model is studied. It is found that the long-run prices in the alternating-move model are higher than for the static model. These long-run prices grow with the discount factor, since the sellers become more patient.

The base model is extended by the introduction of exogenous demand shocks. Then finding an analytic solution using the analysis of Maskin and Tirole (1987) is not possible. Therefore the MPE is calculated numerically. For different values of the shock probability, the margins in which long-run prices fluctuate are determined. The prices set when demand is 
low are always higher than when demand is high. Thus, our findings would support a notion of counter cyclical pricing with respect to the state of demand.

In most of the literature referred to in the introduction, comparable models are considered where besides exogenous timing of decision also the situation is studied wherein the timing is endogenous in combination with adjustment costs. For the endogenous timing situation similar results were found as in the exogenous timing counterparts. Application of endogenous timing in combination with the presence of adjustment costs will also not change any of the results obtained here.

Introducing demand shocks into an infinite-horizon alternating-move homogenous Cournot model leads to the same insights. With respect to quantities the findings are mirrored since they are strategical substitutes.

\section{References}

1. Baye, R.B. and S.F. Ueng (1999), "Commitment and Price Competition in a Dynamic Differentiated-Product Duopoly," Journal of Economics, 69, 41-52.

2. Cyert, R.M. and M.H. DeGroot (1970), "Multiperiod Decision Models with Alternating Choice as the Solution to the Duopoly Problem," Quarterly Journal of Economics, 84, 410-429.

3. De Fraja, G. (1993), "Staggered vs. Synchronized Wage Setting in Oligopoly," European Economic Review, 37, 1507-1522.

4. Eaton, J. And M. Engers (1990), "Intertemporal Price Competition," Econometrica, 58, 637-659.

5. Eckert, A. (2004), "An Alternating-Move Price-Setting Duopoly Model with Stochastic Costs," International Journal of Industrial Organization, 22, 997-1015.

6. Herings, P.J.J. And R.J.A.P. Peeters (2004), "Stationary Equilibria in Stochastic Games: Structure, Selection and Computation," Journal of Economic Theory, 118, $32-60$.

7. Hotelling, H. (1929), "Stability in Competition," Economic Journal, 39, 41-57.

8. LAU, S.-H.P. (2002), "Solution of Multi-Player Linear-Quadratic Alternating-Move Games and Its Application to the Timing Pattern of Wage Adjustment," Computational Economics, 19, 341-357.

9. Maskin, E. And J. Tirole (1987), "A Theory of Dynamic Oligopoly, III: Cournot Competition," European Economic Review, 31, 947-968. 
10. Maskin, E. And J. Tirole (1988a), "A Theory of Dynamic Oligopoly, I: Overview and Quantity Competition with Large Fixed Costs," Econometrica, 56, 549-569.

11. Maskin, E. And J. Tirole (1988b), "A Theory of Dynamic Oligopoly, II: Price Competition, Kinked Demand Curves, and Edgeworth Cycles," Econometrica, 56, 571-599.

12. Maskin, E. And J. Tirole (2001), "Markov Perfect Equilibrium, I: Observable Actions," Journal of Economic Theory, 100, 191-219.

13. McKelvey, R.D. And T.R. Palfrey (1995), "The Holdout Game: An Experimental Study of an Infinitely Repeated Game with Two-Sided Incomplete Information," in: W. Barnett, H. Moulin, M. Salles and N. Schofield (eds.), Social Choice, Welfare, and Ethics, Proceedings of the 8th International Symposium in Economic Theory and Econometrics, Cambridge University Press, Cambridge, pp.321-49.

14. RAth, K.P. (1998), "Stationary and Nonstationary Strategies in Hotelling's Model of Spatial Competition with Repeated Pricing Decisions," International Journal of Game Theory, 27, 525-537.

\section{A Analytic solution of the base model}

Except from a slight different style of exposition, the proofs in this appendix are similar to the proofs of Maskin and Tirole (1987).

We are interested in pairs of dynamic reaction functions $\left(R^{A}, R^{B}\right)$ that form an MPE. In order to show that a pair of dynamic reaction functions forms an MPE, it is enough to rule out profitable one-shot deviations (see Herings and Peeters (2004) for an explanation of the one-deviation property). Hence, $\left(R^{A}, R^{B}\right)$ is an MPE if and only if there exist value functions $\left(\left(V^{A}, W^{A}\right),\left(V^{B}, W^{B}\right)\right)$ such that for any pair of prices $\left(p^{A}, p^{B}\right)$ :

$$
\begin{aligned}
& V^{A}\left(p^{B}\right)=\max _{p}\left\{\pi^{A}\left(p, p^{B}\right)+\delta \cdot W^{A}(p)\right\} \\
& R^{A}\left(p^{B}\right) \in \operatorname{argmax}_{p}\left\{\pi^{A}\left(p, p^{B}\right)+\delta \cdot W^{A}(p)\right\} \\
& W^{A}\left(p^{A}\right)=\pi^{A}\left(p^{A}, R^{B}\left(p^{A}\right)\right)+\delta \cdot V^{A}\left(R^{B}\left(p^{A}\right)\right)
\end{aligned}
$$

and similar expressions for vendor $B$ 's value functions and dynamic reaction function. Here $V^{A}\left(p^{B}\right)$ is vendor $A$ 's present discounted profit if it is about to move, the other vendor's price is $p^{B}$ and the vendors use $\left(R^{A}, R^{B}\right)$ forever; $W^{A}\left(p^{A}\right)$ is vendor $A$ 's present discounted profit if vendor $B$ is about to move and when vendor $A$ is currently committed to price $p^{A}$ and the vendors continue with strategies $R^{A}$ and $R^{B}$ stationarily.

Theorem A.1 When they exist, the dynamic reaction functions are upward sloping. 
Proof Assume, to the contrary, that $p^{A}>\bar{p}^{A}$ and $R^{B}\left(p^{A}\right)<R^{B}\left(\bar{p}^{A}\right)$. By definition, $R^{B}\left(p^{A}\right)$ is a best response to $p^{A}$, i.e.

$$
\pi^{B}\left(p^{A}, R^{B}\left(\bar{p}^{A}\right)\right)+\delta \cdot W^{B}\left(R^{B}\left(\bar{p}^{A}\right)\right) \leq \pi^{B}\left(p^{A}, R^{B}\left(p^{A}\right)\right)+\delta \cdot W^{B}\left(R^{B}\left(p^{A}\right)\right)
$$

and $R^{B}\left(\bar{p}^{A}\right)$ is a best response to $\bar{p}^{A}$, i.e.

$$
\pi^{B}\left(\bar{p}^{A}, R^{B}\left(p^{A}\right)\right)+\delta \cdot W^{B}\left(R^{B}\left(p^{A}\right)\right) \leq \pi^{B}\left(\bar{p}^{A}, R^{B}\left(\bar{p}^{A}\right)\right)+\delta \cdot W^{B}\left(R^{B}\left(\bar{p}^{A}\right)\right)
$$

Combining these two inequalities we find that

$$
\pi^{B}\left(p^{A}, R^{B}\left(\bar{p}^{A}\right)\right)-\pi^{B}\left(p^{A}, R^{B}\left(p^{A}\right)\right)+\pi^{B}\left(\bar{p}^{A}, R^{B}\left(p^{A}\right)\right)-\pi^{B}\left(\bar{p}^{A}, R^{B}\left(\bar{p}^{A}\right)\right) \leq 0
$$

which is equivalent to

$$
\int_{\bar{p}^{A}}^{p^{A}} \int_{R^{B}\left(p^{A}\right)}^{R^{B}\left(\bar{p}^{A}\right)} \frac{\partial^{2}}{\partial x \partial y} \pi^{B}(x, y) \mathrm{d} y \mathrm{~d} x \leq 0 .
$$

But $\frac{\partial^{2}}{\partial x \partial y} \pi^{B}(x, y)=\frac{1}{2 \tau}>0$. We have a contradiction.

The first-order condition for the optimization problem is

$$
\frac{\partial}{\partial x} \pi^{A}\left(R^{A}\left(p^{B}\right), p^{B}\right)+\delta \cdot \frac{\mathrm{d}}{\mathrm{d} p} W^{A}\left(R^{A}\left(p^{B}\right)\right)=0 .
$$

Since $p^{A}=R^{A}\left(p^{B}\right)$, we have

$$
\frac{\partial}{\partial x} \pi^{A}\left(p^{A},\left(R^{A}\right)^{-1}\left(p^{A}\right)\right)+\delta \cdot \frac{\mathrm{d}}{\mathrm{d} p} W^{A}\left(p^{A}\right)=0,
$$

and since $p^{B}=R^{B}\left(p^{A}\right)$, we have

$$
\text { (2) } \quad \frac{\partial}{\partial x} \pi^{A}\left(R^{A}\left(R^{B}\left(p^{A}\right)\right), R^{B}\left(p^{A}\right)\right)+\delta \cdot \frac{\mathrm{d}}{\mathrm{d} p} W^{A}\left(R^{A}\left(R^{B}\left(p^{A}\right)\right)\right)=0 .
$$

Moreover, from the maximization problem we can formulate the following Bellman equation

$$
W^{A}\left(p^{A}\right)=\pi^{A}\left(p^{A}, R^{B}\left(p^{A}\right)\right)+\delta \cdot \pi^{A}\left(R^{A}\left(R^{B}\left(p^{A}\right)\right), R^{B}\left(p^{A}\right)\right)+\delta^{2} \cdot W^{A}\left(R^{A}\left(R^{B}\left(p^{A}\right)\right)\right) .
$$

Differentiation of the Bellman equation gives

$$
\begin{aligned}
\frac{\mathrm{d}}{\mathrm{d} p} W^{A}\left(p^{A}\right)= & \frac{\partial}{\partial x} \pi^{A}\left(p^{A}, R^{B}\left(p^{A}\right)\right)+\frac{\partial}{\partial y} \pi^{A}\left(p^{A}, R^{B}\left(p^{A}\right)\right) \cdot \frac{\mathrm{d}}{\mathrm{d} p^{A}} R^{B}\left(p^{A}\right) \\
& +\delta \cdot \frac{\partial}{\partial x} \pi^{A}\left(R^{A}\left(R^{B}\left(p^{A}\right)\right), R^{B}\left(p^{A}\right)\right) \cdot \frac{\mathrm{d}}{\mathrm{d} p^{B}} R^{A}\left(R^{B}\left(p^{A}\right)\right) \cdot \frac{\mathrm{d}}{\mathrm{d} p^{A}} R^{B}\left(p^{A}\right) \\
& +\delta \cdot \frac{\partial}{\partial y} \pi^{A}\left(R^{A}\left(R^{B}\left(p^{A}\right)\right), R^{B}\left(p^{A}\right)\right) \cdot \frac{\mathrm{d}}{\mathrm{d} p^{A}} R^{B}\left(p^{A}\right) \\
& +\delta^{2} \cdot \frac{\mathrm{d}}{\mathrm{d} p} W^{A}\left(R^{A}\left(R^{B}\left(p^{A}\right)\right)\right) \cdot \frac{\mathrm{d}}{\mathrm{d} p^{B}} R^{A}\left(R^{B}\left(p^{A}\right)\right) \cdot \frac{\mathrm{d}}{\mathrm{d} p^{A}} R^{B}\left(p^{A}\right) .
\end{aligned}
$$

Substitution of (2) gives

$$
\begin{aligned}
\frac{\mathrm{d}}{\mathrm{d} p} W^{A}\left(p^{A}\right)= & \frac{\partial}{\partial x} \pi^{A}\left(p^{A}, R^{B}\left(p^{A}\right)\right)+\frac{\partial}{\partial y} \pi^{A}\left(p^{A}, R^{B}\left(p^{A}\right)\right) \cdot \frac{\mathrm{d}}{\mathrm{d} p^{A}} R^{B}\left(p^{A}\right) \\
& +\delta \cdot \frac{\partial}{\partial y} \pi^{A}\left(R^{A}\left(R^{B}\left(p^{A}\right)\right), R^{B}\left(p^{A}\right)\right) \cdot \frac{\mathrm{d}}{\mathrm{d} p^{A}} R^{B}\left(p^{A}\right)
\end{aligned}
$$


and subsequent substitution of (1) gives

$$
\begin{aligned}
-\frac{1}{\delta} \cdot \frac{\partial}{\partial x} \pi^{A}\left(p^{A},\left(R^{A}\right)^{-1}\left(p^{A}\right)\right)= & \frac{\partial}{\partial x} \pi^{A}\left(p^{A}, R^{B}\left(p^{A}\right)\right)+\frac{\partial}{\partial y} \pi^{A}\left(p^{A}, R^{B}\left(p^{A}\right)\right) \cdot \frac{\mathrm{d}}{\mathrm{d} p^{A}} R^{B}\left(p^{A}\right) \\
& +\delta \cdot \frac{\partial}{\partial y} \pi^{A}\left(R^{A}\left(R^{B}\left(p^{A}\right)\right), R^{B}\left(p^{A}\right)\right) \cdot \frac{\mathrm{d}}{\mathrm{d} p^{A}} R^{B}\left(p^{A}\right) .
\end{aligned}
$$

The latter expression can be simplified to

$$
\frac{\mathrm{d}}{\mathrm{d} p^{A}} R^{B}\left(p^{A}\right)=\frac{-\frac{\partial}{\partial x} \pi^{A}\left(p^{A},\left(R^{A}\right)^{-1}\left(p^{A}\right)\right)-\delta \cdot \frac{\partial}{\partial x} \pi^{A}\left(p^{A}, R^{B}\left(p^{A}\right)\right)}{\delta \cdot \frac{\partial}{\partial y} \pi^{A}\left(p^{A}, R^{B}\left(p^{A}\right)\right)+\delta^{2} \cdot \frac{\partial}{\partial y} \pi^{A}\left(R^{A}\left(R^{B}\left(p^{A}\right)\right), R^{B}\left(p^{A}\right)\right)} .
$$

Next, we look for linear dynamic reaction functions:

$$
R^{A}\left(p^{B}\right)=\alpha^{A}+\beta^{A} p^{B} \quad \text { and } \quad R^{B}\left(p^{A}\right)=\alpha^{B}+\beta^{B} p^{A} .
$$

Moreover, we know that

$$
\frac{\partial}{\partial p^{A}} \pi^{A}=\frac{1}{2}+\frac{p^{B}-2 p^{A}}{2 \tau} \quad \text { and } \quad \frac{\partial}{\partial p^{B}} \pi^{A}=\frac{p^{A}}{2 \tau} .
$$

Therefore condition (3) boils down to

$$
\beta^{B}=\left(-\left[\frac{1}{2}+\frac{\left(p^{A}-\alpha^{A}\right) / \beta^{A}-2 p^{A}}{2 \tau}\right]-\delta \cdot\left[\frac{1}{2}+\frac{\alpha^{B}+\beta^{B} p^{A}-2 p^{A}}{2 \tau}\right]\right) /\left(\delta \cdot \frac{p^{A}}{2 \tau}+\delta^{2} \cdot \frac{\alpha^{A}+\beta^{A}\left(\alpha^{B}+\beta^{B} p^{A}\right)}{2 \tau}\right)
$$

or equivalently

$$
\begin{aligned}
& {\left[\delta^{2}\left(\beta^{A}\right)^{2}\left(\beta^{B}\right)^{2}+2 \delta \beta^{A} \beta^{B}-2(1+\delta) \beta^{A}+1\right] p^{A}=} \\
& \quad-\delta^{2} \alpha^{B}\left(\beta^{A}\right)^{2} \beta^{B}-\delta^{2} \alpha^{A} \beta^{A} \beta^{B}-\delta \alpha^{B} \beta^{A}+\alpha^{A}-(1+\delta) \beta^{A} \tau .
\end{aligned}
$$

As a constant times $p^{A}$ is constant, the first constant must be zero, i.e.

$$
\delta^{2}\left(\beta^{A}\right)^{2}\left(\beta^{B}\right)^{2}+2 \delta \beta^{A} \beta^{B}-2(1+\delta) \beta^{A}+1=0 .
$$

By symmetry we also have

$$
\delta^{2}\left(\beta^{A}\right)^{2}\left(\beta^{B}\right)^{2}+2 \delta \beta^{A} \beta^{B}-2(1+\delta) \beta^{B}+1=0 .
$$

From these two equations it is clear that $\beta^{A}=\beta^{B}=\beta$. Hence, we can drop the superscripts:

$$
\delta^{2} \beta^{4}+2 \delta \beta^{2}-2(1+\delta) \beta+1=0 .
$$

This equation has four solutions of which two of them are real. One of these lies in the interval $\left(0, \frac{1}{2}\right)$ and the other in the interval $\left(\frac{1}{\sqrt{\delta}}, \frac{1}{\delta}\right)$. The root in the second interval can be shown to give rise to a dynamically unstable path (see Maskin and Tirole (1987)). The root in the first interval is relevant for the present purpose. This root leads to dynamic reaction functions for which there is a steady-state price and that are therefore dynamically stable.

But, also the right-hand side of (4) has to be equal to zero, i.e.

$$
\delta^{2} \beta^{3} \alpha^{B}+\delta^{2} \beta^{2} \alpha^{A}+\delta \beta \alpha^{B}-\alpha^{A}=-(1+\delta) \beta \tau,
$$

and again by symmetry

$$
\delta^{2} \beta^{3} \alpha^{A}+\delta^{2} \beta^{2} \alpha^{B}+\delta \beta \alpha^{A}-\alpha^{B}=-(1+\delta) \beta \tau .
$$


Combining these two equalities gives

$$
\left(\delta^{2} \beta^{3}-\delta^{2} \beta^{2}+\delta \beta+1\right)\left(\alpha^{B}-\alpha^{A}\right)=0
$$

from which we see that $\alpha^{A}=\alpha^{B}$ and again we can drop the superscripts:

$$
\delta^{2} \beta^{3} \alpha+\delta^{2} \beta^{2} \alpha+\delta \beta \alpha-\alpha=-(1+\delta) \beta \tau .
$$

Solving this equation for $\alpha$ gives

$$
\alpha=\frac{-(1+\delta) \tau \beta}{\delta^{2} \beta^{3}+\delta^{2} \beta^{2}+\delta \beta-1} .
$$

Theorem A.2 For any discount factor $\delta$ : (1) there exists a unique linear MPE; (2) this MPE is dynamically stable; (3) each vendor's steady state price is equal to $\bar{p}=\frac{\alpha}{1-\beta}$, is equal to the static Hotelling solution for $\delta=0$, and grows with the discount factor.

Proof (1) Since the dynamic reaction functions are linear and the profit functions quadratic, the valuation functions are quadratic. From this it is easily found that the objection function is concave. This means that the first-order conditions are not only necessary but also sufficient. All candidate linear MPEs satisfy the fourth degree polynomial equation that determines $\beta$. But, only the dynamics associated with one of the roots is consistent with an MPE. Thus, the symmetric pair of dynamic reaction functions that are determined by $\alpha$ and the $\beta$ above form a unique linear MPE.

(2) Dynamic stability is obtained by the slope of the dynamic reaction curves that have a slope larger than 0 and less than $\frac{1}{2}$ and thus less than 1 in absolute value.

(3) The behavior of the equilibrium dynamic reaction functions and of the steady state are subject in Sections 4 and 5 . 